\title{
FIGO/TNM Stage
}

National Cancer Institute

\section{Source}

National Cancer Institute. FIGO/T NM Stage. NCI Thesaurus. Code C157232.

A staging system that involved either the FIGO (International Federation of Gynecology and Obstetrics) and/or the TNM system. 